\title{
Three-dimensional printing models in congenital heart disease education for medical students: a controlled comparative study
}

Wei Su${ }^{1}$, Yunbin $\mathrm{Xiao}^{2}$, Siping $\mathrm{He}^{3}$, Peng Huang ${ }^{2}$ and Xicheng Deng ${ }^{2^{*}}$

\begin{abstract}
Background: This study sought to assess, using subjective (self-assessment) and objective (MCQ) methods, the efficacy of using heart models with ventricular septal defect lesions produced with three-dimensional printing technology in a congenital heart disease curriculum for medical students.

Methods: Three computed tomography datasets of three subtypes of ventricular septal defects (perimembranous, subarterial and muscular, one for each) were obtained and processed for building into and printing out 3D models. Then a total of 63 medical students in one class were randomly allocated to two groups (32 students in the experimental, and 31 the control). The two groups participated in a seminar with or without a 3D heart model, respectively. Assessment of this curriculum was carried out using Likert-type questionnaires as well as an objective multiple choice question test assessing both knowledge acquisition, and structural conceptualization. Open-ended questions were also provided for getting advice and suggestion on 3D model utilization in CHD education.

Results: With these 3D models, feedback shown in the questionnaires from students in experimental group was significantly more positive than their classmates in the control. And the test results also showed a significant difference in structural conceptualization in favor of the experimental group.

Conclusion: It is effective to use heart models created using current 3D printing technology for congenital heart disease education. It stimulates students' interest in congenital heart disease and improves the outcomes of medical education.
\end{abstract}

Keywords: Medical education, Three-dimensional printing, Congenital heart disease

\section{Background}

Three-dimensional printing, a kind of rapid prototyping and manufacturing technology, has been widely utilized in medicine [1-3]. Applications of 3D printing technology in the medical field includes surgical guidance during surgery, preoperative procedural planning [4-7] as well as management of difficult clinical situations [8]. Medical education has also witnessed its wide application [9-12]. Simulation-based training and education with 3D models for students (including nursing, nursing

\footnotetext{
* Correspondence: justindxc@gmail.com

${ }^{2}$ Heart Center, Hunan Children's Hospital, No. 86 Ziyuan Road, Changsha 410007, China

Full list of author information is available at the end of the article
}

anesthesia, and medical trainees) has been reported to improve understanding of medical knowledge and clinical outcomes [13-15].

Congenital heart defects (CHD) is a group of common defects with a prevalence of $0.8-1.2 \%$ of all live birth [16]. A clinical understanding of CHD is crucial as many of the anomalies can be life threatening. The traditional educational approach to teaching CHD involves the use of pathological specimens, off-the-shelf normal heart anatomic models made of plastics, medical imaging data(including CT, MR and Echocardiography), as well as textbooks. This approach is difficult to conceptualize and visualize for medical students considering the variability of all the different types of CHDs [17]. Since every 
single CHD case is unique and different in terms of their anatomy and pathophysiology, this poses a huge challenge for learners.

The recent advancement and popularization of three-dimensional (3D) printing has made it possible to create high-fidelity heart models with complex cardiac lesions from source imaging data [18]. Theoretically, 3D printed models have better spatial and structural visualization and can be used as didactic tools for better understanding of complex heart or vessel anatomy as well as easier explanation of abnormal anatomical heart and vessel structures. Some previous studies have shown its effectiveness of using 3D-printed models in CHD curriculum [11, 18, 19]. However, no controlled study with objective evaluation methods has ever been performed. The non-randomized controlled nature and subjective assessment in previous studies made the results less convincing. Therefore, we carried out this randomized controlled study to verify the efficacy of 3D printed heart model use in medical education.

\section{Methods}

Computed tomography imaging (CT) data were extracted and 3D printing process was performed to produce lesion-specific models. In detail, CT data of three ventricular septal defects (VSD) (subarterial, membranous, and muscular types, one for each) were exported from institutional image archive system and then imported and analyzed. After post-process, the reconstructed images underwent $3 \mathrm{D}$ volume rendering to create a digital heart model. Following optimization and simplification, final data were generated and sent to SL600 3D printer (ZRapid Tech, Wujiang, China) for model printing. High-fidelity plastic heart models were successfully produced one for each of three common ventricular septal defect subtypes (subarterial, membranous, and muscular types) (Figs. 1 and 2).

Curriculum incorporating these 3D heart models was then developed. Besides the 3D prints, compulsory CHD syllabus involving relevant knowledge on anatomy and classification, pathophysiology, clinical findings, workups, clinical management and prognosis of ventricular septal defects for medical students was included in the curriculum and then used in seminars. The curriculum developer, who was also the lecturer to hold the seminars, was an associate professor of pediatrics with more than 10 years of teaching experience of lectures and seminars for medical students and medical graduates.

A class of 63 medical students was chosen to carry out the study. They were informed beforehand about this study and agreed to participate. They were randomly allocated to two groups, 32 students in the experimental and 31 in the control, using computerized random number generation method. All the subjects participated in one of two teaching seminars by the same lecturer. The

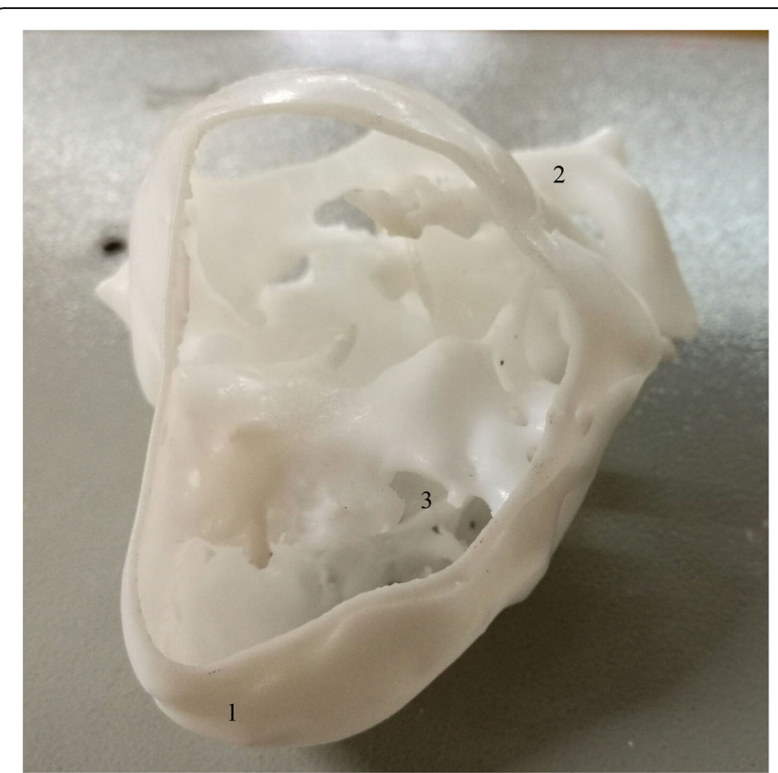

Fig. 1 Front view of a model. 1. apex; 2. aortic arch; 3. ventricular septal defect

inter-group demographics were compared and showed no statistical difference. Instead of pre-seminar assessment, we used latest academic test score as baseline academic performance (Table 1).

Seminars were designed and structured for the two groups. For the experimental group, the seminar consisted of two consecutive components: (1) a succinct introduction to $3 \mathrm{D}$ printing technology and its use in medical education and practice; (2) a didactic session on ventricular defects integrating $3 \mathrm{D}$ models in teaching subtypes of VSD, specifically, in its classification and

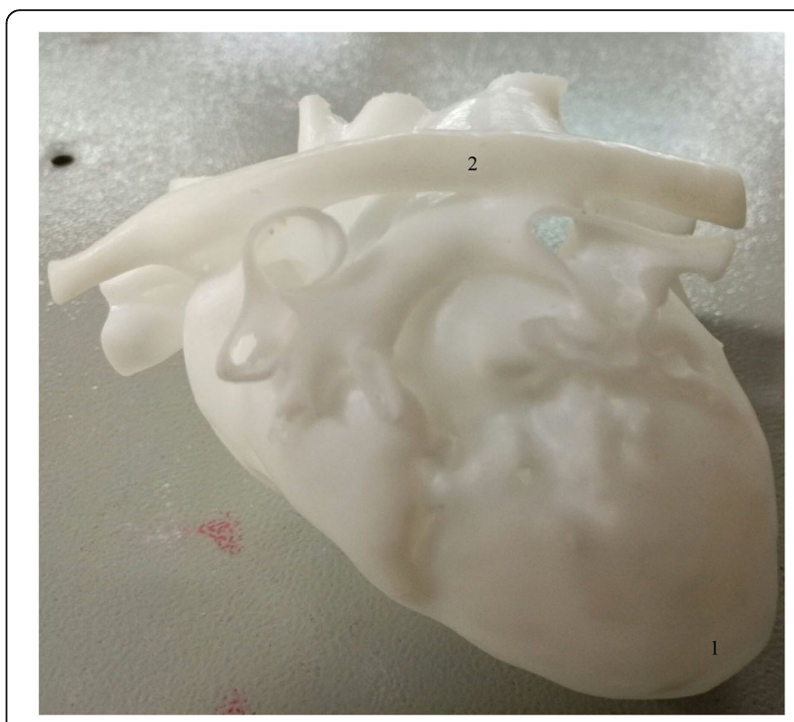

Fig. 2 Back view of the same model as shown in Fig. 1. 1. apex; 2. descending aorta 
Table 1 Intergroup comparison of demographics and academic performance

\begin{tabular}{llll}
\hline Group demographics & Experimental & Control & $P$ Value \\
\hline Gender(M/F) (total number) & $16 / 16(32)$ & $14 / 17(31)$ & 0.802 \\
Age & $21.00 \pm 0.57$ & $21.06 \pm 0.58$ & 0.655 \\
Academic performance & $73.43 \pm 10.84$ & $76.68 \pm 10.93$ & 0.242 \\
\hline
\end{tabular}

pathophysiology. Yet for the control subjects, they attended a seminar with only the second component but without 3D models (only with images and animations) (Table 2). The teaching method and curriculum in the control group had been used for years and approved by teachers and students, so the participants in control group were not regarded as being at a disadvantage of learning.

At the end of each seminar, all participants completed a subjective 1-10 scale Likert-type questionnaire and a prepared objective multiple choice tests (MCQ). The questionnaires were identical between the two groups. The questionnaire included 10 items that examined two main educational components which were knowledge acquisition (including clinical presentations and pathophysiology, 3 items) and structural conceptualization (including classification/types and anatomy, 2 items) as well as 5 items regarding students' appraisal of the seminars and 3D prints. Each item got a score ranging 1-10 and the total score of the two main educational components for each participant were summed up. The MCQs included 10 items on the anatomy, pathophysiology and scenario cases of VSD. The same MCQs had been used as standard test after didactic session for VSD in the late several years before the study to assess the students' grasp of knowledge about CHD. The questionnaire as well as the MCQ was developed by one of the authors who was the only teacher involved in the seminars. The MCQs and questionnaire papers were marked with personal information for recording each student's academic performance. Then the data were de-identified for use in this study. All original documents were kept on file in case of a review.

Data was statistically presented and processed as appropriate. Shapiro-Wilk test was used to confirm if it was normally distributed where necessary. Statistical analysis was then conducted using the Chi-square and Student's T test as appropriate; two-tailed $P$ values of 0.05 were used as indicators of significance. Two open-ended questions were also provided in the experimental group for advice on 3D model utilization in CHD education in order for improvement in the future. The focuses of the answers were extracted manually and accumulated as frequencies. The original version and translated MCQ paper, questionnaire and open-ended questions have been uploaded as Additional files 1, 2, 3.

\section{Results}

There was no difference in gender, age and academic performance between the groups (Table 1). At the end of each seminar, the questionnaire results showed that students in the experimental group reported significant improvement in VSD learning and better seminar outcomes $(P<.0001)$ (Table 3$)$. The test results were statistically significant in favor of the experimental group $(P=0.02)$. The inter-group differences of both questionnaire and test results were statistically significant in structural conceptualization ( $P=0.02,0.03$, respectively), but not in knowledge acquisition $(P=0.09,0.06$, respectively).

Data from open-ended questions included in the questionnaires in experimental group showed advantages of using 3D models over traditional teaching method and aspects that need to be improved. On the one hand, a total of $27(84.4 \%)$ students reported better understanding of heart anatomy, followed by $16(50.0 \%)$ admitted a positive interest in cardiology and cardiac surgery as show in the results of question 1 . On the other hand, in question 2 , as high as $18(56.2 \%)$ subjects reported a need for improvement in cardiac structures especially intra-cardiac ones exemplified by the valves and their apparatuses in the 3D prints. Similar to that was a complaint about lack of explicit delineation of structures by $11(34.4 \%)$ students.

\section{Discussion}

In the present study, our finding has shown overall improvement and better structural conceptualization in

Table 2 VSD cirriculum in the two groups

\begin{tabular}{lll}
\hline Cirriculum structure & Experimental(minutes) & Control(minutes) \\
\hline Introduction to 3D printing technology & 5 & N/A \\
Anatomy and classification & 5 (3D prints used) & 5 \\
Pathophysiology & $15(3 \mathrm{D}$ prints used) & 15 \\
Clinical findings & 3 & 3 \\
Workups & 3 & 3 \\
Clinical management and prognosis & 4 & 4 \\
Total seminar duration & 35 & 30 \\
After-seminar test and questionaire & 25 & 25 \\
\hline
\end{tabular}


Table $\mathbf{3}$ Tests and questionnaire results

\begin{tabular}{llll}
\hline Test / appraisal(score) $^{\text {a }}$ & Experimental & Control & $P$ Value \\
\hline Test score(100) & $62.50 \pm 19.04$ & $51.29 \pm 17.55$ & 0.02 \\
$\quad$ Structural conceptualization (30) & $18.44 \pm 6.67$ & $14.52 \pm 7.11$ & 0.03 \\
$\quad$ Knowledge acquisition (70) & $44.06 \pm 16.37$ & $36.77 \pm 12.54$ & 0.06 \\
Likert-type questionnaire (100) $^{\text {b }}$ & $72.19 \pm 14.91$ & $56.12 \pm 10.55$ & $<0.0001$ \\
Structural conceptualization (20) & $15.50 \pm 2.22$ & $14.16 \pm 2.17$ & 0.02 \\
$\quad$ Knowledge acquisition (30) & $22.63 \pm 3.00$ & $21.39 \pm 2.50$ & 0.09 \\
\hline
\end{tabular}

a each item has a full score of 10

${ }^{b}$ the questionnaire contains other 5 items that are not included in the table

sub-analysis with both test scores and questionnaire following a 3D heart model assisted seminar.

The major difference of this study from previous ones [18] is both MCQ and self-assessment were used in the evaluation of the simulation based teaching and a randomized controlled study designed was used. In previous studies [20], the subjective methods used may have potential statistical bias and warranted a case-controlled study. While our result confirmed the conclusion from previous studies, the comparative nature of this study made it more convincing, though we used previous academic test score as a baseline instead of pre-seminar test. We did not use MCQs before the seminars for fear that it may bring bias if the same test was used both before and after the seminars. According to our results, the students had no inter-group difference in academic performance. (Table 1).

Though every single case with CHD is regarded unique, we only printed out three models representing different types of VSD. First, this is a pilot study to verify it's efficacy in medical student education. Second, for medical education purpose, we thought it was enough to use three typical models to convey sufficient information to students, rather than in a clinical setting that every single case might seem different with regard to anatomy of a VSD.

As for the 3D printing technology and the imaging source and resolution of printed models, though some specific structures especially intra-cardiac ones, including heart valves and trabecular muscles, needed to be improved in fidelity, the CT dataset proved to be a sufficient source to create ventricular septal defect models with the aid of relevant softwares and 3D printing technology [21]. The shortcomings in detailing intra-cardiac anatomy are not because of limitation of the 3D printing per se, but for limited resolution of source images. Computed tomography (CT) images and cardiac magnetic resonance imaging (MRI) were used as source data for printing the high-fidelity models. Compared to MRI, CT provides superior spatial resolution [22] but radiation is an inevitable shortage. Both of them as image source are limited and some researchers [11] have developed an approach to utilizing echocardiographic images as source data to enhance fidelity of heart models, especially for valve anatomy.

The use of 3D productions in medical education have several advantages: first, comparing to cadaver, it is much cheaper which may in turn alleviate the financial, ethical, cultural, and logistical difficulties of maintaining a cadaver-based curriculum [12]; second, comparing to pictorial images, diagrams, and conventional echocardiography, which are all two-dimensional hence conceptually challenging for beginning learners, It gives a true spatial relationship to allow tangible manipulation of the extra- and intra-cardiac structures [23]. Third, comparing to anatomical off-the-shelf models, even those of high quality are rather schematic and do not show the range of variation present in different human populations in health and disease $[24,25]$. The anatomical advantage of using $3 \mathrm{D}$ prints was confirmed by our results in both test and questionnaire. Also, the results from open-ended questions showed an increased interest of the students in cardiology and cardiac surgery.

However, there were some limitations in the study. First, for the statistical aspects, the subject number was relatively small and randomization was undertaken in a preexisting class. And we did not test the students prior to the seminar. Instead, we used a latest academic score for baseline comparison. As such, there may remain some biases in the study. Second, as mentioned before, these 3D prints are not without drawbacks. Currently, limited to image quality, it remains a challenge to accurately print out intra-cardiac structures, making its application confined to specific types of CHD.

As mentioned above, 3D printed models are generally regarded to advantage over other media in medical education for its superior spatial and structural visualization and patient-specific features. This is also demonstrated by the present study results, especially better structural conceptualization in sub-analysis results. Further application in CHD education may include multiple disease including atrial septal defect, tetralogy of Fallot, etc. In the near future, 3D printing technologies are expected to advance to make more accurate heart prints, especially intra-cardiac structures including valves and their apparatus. 


\section{Conclusions}

In this study we have demonstrated the efficacy of incorporating a 3D printing heart model into a medical curriculum about CHD. We have used both MCQ and self-assessment methods in this comparative study and demonstrated that better structural conceptualization by students were achieved. As 3D printing continues to advance and simulation-based education becomes more extensively utilized, this novel technology is expected to broadly apply in the education of congenital heart defects.

\section{Additional files}

Additional file 1: Questionnaire and quiz scores. (XLSX $13 \mathrm{~kb}$ )

Additional file 2: Quiz MCQs and translated stems. (DOC 29 kb)

Additional file 3: Original questionnaires and English translation. (DOC 27 kb)

\section{Abbreviations}

3D: Three dimensional; CHD: Congenital heart defects; CT: Computed tomography imaging; MCQ: Multiple choice question/test; MRI: Magnetic resonance imaging; VSD: Ventricular septal defect

\section{Funding}

This project was supported by the Education Department of Hunan Province (Grant No. 2016-400-744) and the National Natural Sciences Foundation of China (Grant No. 81500041).

\section{Availability of data and materials}

Uploaded within submission.

\section{Authors' contributions}

WS: carrying out study and data collection. YX: study design and carrying out study. SH: three-dimensional prints process. PH: study design. XD: study design, initial draft writing and data analysis. All authors contributed significantly to subsequent major revisions and agreed to give final approval of the version to be submitted in its present form.

\section{Ethics approval and consent to participate}

This study was approved by the Institutional Review Board at the Hospital Affiliated with Xiangnan University School of Medicine in the form of modified teaching method study. Considering the nature of the study, written consent from each individual participating in the study was waived by the IRB.

\section{Consent for publication}

Since this was a teaching method study without patients involved, consent to publish was waived by the institutional review board.

\section{Competing interests}

The authors declare that they have no competing interests.

\section{Publisher's Note}

Springer Nature remains neutral with regard to jurisdictional claims in published maps and institutional affiliations.

\section{Author details}

${ }^{1}$ Research Unit for Pediatrics, Xiangnan University School of Medicine, Chenzhou 423000, China. ${ }^{2}$ Heart Center, Hunan Children's Hospital, No. 86 Ziyuan Road, Changsha 410007, China. ${ }^{3}$ Department of Radiology, Hunan Children's Hospital, Changsha 410007, China.
Received: 9 April 2018 Accepted: 25 July 2018

Published online: 02 August 2018

\section{References}

1. Michalski MH, Ross JS. The shape of things to come: 3D printing in medicine. Jama. 2014;312(21):2213-4

2. Tuomi J, Paloheimo KS, Vehvilainen J, Bjorkstrand R, Salmi M, Huotilainen E, Kontio R, Rouse S, Gibson I, Makitie AA. A novel classification and online platform for planning and documentation of medical applications of additive manufacturing. Surg Innov. 2014;21(6):553-9.

3. Malik HH, Darwood AR, Shaunak S, Kulatilake P, El-Hilly AA, Mulki O, Baskaradas A. Three-dimensional printing in surgery: a review of current surgical applications. J Surg Res. 2015;199(2):512-22.

4. Kurenov SN, lonita C, Sammons D, Demmy TL. Three-dimensional printing to facilitate anatomic study, device development, simulation, and planning in thoracic surgery. J Thorac Cardiovasc Surg. 2015;149(4):973-9. e971

5. Chae MP, Rozen WM, McMenamin PG, Findlay MW, Spychal RT, HunterSmith DJ. Emerging Applications of Bedside 3D Printing in Plastic Surgery. Front Surg. 2015;2:25.

6. Youssef RF, Spradling K, Yoon R, Dolan B, Chamberlin J, Okhunov Z, Clayman R, Landman J. Applications of three-dimensional printing technology in urological practice. BJU Int. 2015;116(5):697-702.

7. Flugge TV, Nelson K, Schmelzeisen R, Metzger MC. Three-dimensional plotting and printing of an implant drilling guide: simplifying guided implant surgery. J Oral Maxillofac Surg. 2013;71(8):1340-6.

8. Zopf DA, Hollister SJ, Nelson ME, Ohye RG, Green GE. Bioresorbable airway splint created with a three-dimensional printer. New Eng J Med. 2013; 368(21):2043-5.

9. Mahmoud A, Bennett M. Introducing 3-Dimensional Printing of a Human Anatomic Pathology Specimen: Potential Benefits for Undergraduate and Postgraduate Education and Anatomic Pathology Practice. Arch Pathol Lab Med. 2015;139(8):1048-51.

10. AbouHashem Y, Dayal M, Savanah S, Strkalj G. The application of 3D printing in anatomy education. Med Educ Online. 2015;20:29847.

11. Costello JP, Olivieri LJ, Krieger A, Thabit O, Marshall MB, Yoo SJ, Kim PC, Jonas RA, Nath DS. Utilizing Three-Dimensional Printing Technology to Assess the Feasibility of High-Fidelity Synthetic Ventricular Septal Defect Models for Simulation in Medical Education. World J Pediatr Congenit Heart Surg. 2014;5(3):421-6.

12. Lim KH, Loo ZY, Goldie SJ, Adams JW, McMenamin PG. Use of 3D printed models in medical education: A randomized control trial comparing 3D prints versus cadaveric materials for learning external cardiac anatomy. Anat Sci Educ. 2016:9(3):213-21.

13. Paige JT, Garbee DD, Kozmenko V, Yu Q, Kozmenko L, Yang T, Bonanno L, Swartz W. Getting a head start: high-fidelity, simulation-based operating room team training of interprofessional students. J Am Coll Surg. 2014; 218(1):140-9.

14. Ciobotaru V, Combes N, Martin CA, Marijon E, Maupas E, Bortone A,

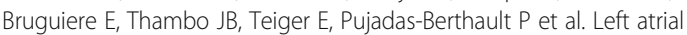
appendage occlusion simulation based on three-dimensional printing: New insights in outcome and technique. Eurolntervention. 2018;14(2):176-84

15. Mashiko T, Otani K, Kawano R, Konno T, Kaneko N, Ito Y, Watanabe E. Development of three-dimensional hollow elastic model for cerebral aneurysm clipping simulation enabling rapid and low cost prototyping. World Neurosurg. 2015;83(3):351-61.

16. Hoffman J. The global burden of congenital heart disease. Cardiovasc J Afr. 2013;24(4):141-5.

17. Valverde I. Three-dimensional Printed Cardiac Models: Applications in the Field of Medical Education, Cardiovascular Surgery, and Structural Heart Interventions. Revista espanola de cardiologia. 2017;70(4):282-91.

18. Costello JP, Olivieri LJ, Su L, Krieger A, Alfares F, Thabit O, Marshall MB, Yoo SJ, Kim PC, Jonas RA, et al. Incorporating three-dimensional printing into a simulation-based congenital heart disease and critical care training curriculum for resident physicians. Congenit Heart Dis. 2015;10(2):185-90.

19. Loke T, Krieger A, Sable C, Olivieri L. Novel Uses for Three-Dimensional Printing in Congenital Heart Disease, vol. 4; 2016

20. Biglino G, Capelli C, Wray J, Schievano S, Leaver LK, Khambadkone S, Giardini A, Derrick G, Jones A, Taylor AM. 3D-manufactured patient-specific models of congenital heart defects for communication in clinical practice: feasibility and acceptability. BMJ open. 2015;5(4):e007165. 
21. Noecker AM, Chen JF, Zhou Q, White RD, Kopcak MW, Arruda MJ, Duncan BW. Development of patient-specific three-dimensional pediatric cardiac models. ASAIO J. 2006;52(3):349-53.

22. Sorensen C, Gach P, Pico H, Hugues N, Dabadie A, Desvignes C, Bourliere B, Aschero A, Colavolpe N, Petit P, et al. Cardiac CT or MRI in pediatric practice: Which one to choose? Diagn Interven Imaging. 2016;97(5):513-7.

23. Mishra S. Application of 3D printing in medicine. Indian Heart J. 2016;68(1): 108-9.

24. Bergman RA. Thoughts on human variations. Clin Anat. 2011;24(8):938-40.

25. Strkalj G, Spocter MA, Wilkinson AT. Anatomy, medical education, and human ancestral variation. Anat Sci Educ. 2011:4(6):362-5.

Ready to submit your research? Choose BMC and benefit from:

- fast, convenient online submission

- thorough peer review by experienced researchers in your field

- rapid publication on acceptance

- support for research data, including large and complex data types

- gold Open Access which fosters wider collaboration and increased citations

- maximum visibility for your research: over $100 \mathrm{M}$ website views per year

At BMC, research is always in progress.

Learn more biomedcentral.com/submissions 